\title{
Evaluation of the driving performance of a surface acoustic wave linear motor
}

\author{
Katsuhiko Asai \\ Matsushita Electric Industrial Co., Ltd., Advanced Technology Research Laboratories, \\ 3-10-1 Higashimita, Tama-ku, Kawasaki 214-8501, Japan \\ Minoru Kuribayashi Kurosawa \\ Tokyo Institute of Technology, Department of Advanced Applied Electronics, \\ 4259 Nagatutamati, Midori-ku, Yokohama 226-8502, Japan \\ Toshiro Higuchi \\ The University of Tokyo, Department of Precision Machinery Engineering, \\ 7-3-1 Hongo, Bunkyo-ku, Tokyo, 113-8656, Japan
}

\begin{abstract}
This paper describes influences of a preload and vibration amplitude on driving performance of a surface acoustic wave (SAW) linear motor, and proposes a simulation model for the SAW linear motor. In experimental results, maximum output force and maximum output power of the SAW linear motor depended on it's pre-load. Optimum pre-loads for the maximum output force and for the maximum output power were both linear functions of the SAW linear motor's vibration amplitude. No-load velocity of the SAW linear motor, however, decreased linearly with increasing pre-load. A new simulation model to explain these phenomena is proposed. There is a good agreement between the experimental and simulation results.
\end{abstract}

\section{INTRODUCTION}

A SAW motor is a linear actuator of friction drive which utilizes the Rayleigh wave. In past researches, operation of the SAW motor using a multi-contactpoint slider or a silicon slider was demonstrated [1], [2]. It has been shown that output force of a SAW motor depends on force (pre-load) which presses a slider against a stator substrate. However, with using the silicon slider, the influence of the pre-load on no-load velocity, and the relation between the optimum pre-load and vibration amplitude have not yet been found out. On the other hand, a simulation model for explaining the influence of the pre-load on driving performance was proposed by Kurosawa et al [1], [3]. However, this conventional simulation model was unable to calculate the optimum pre-load. In the simulation model, dynamic output force is treated as the product of the pre-

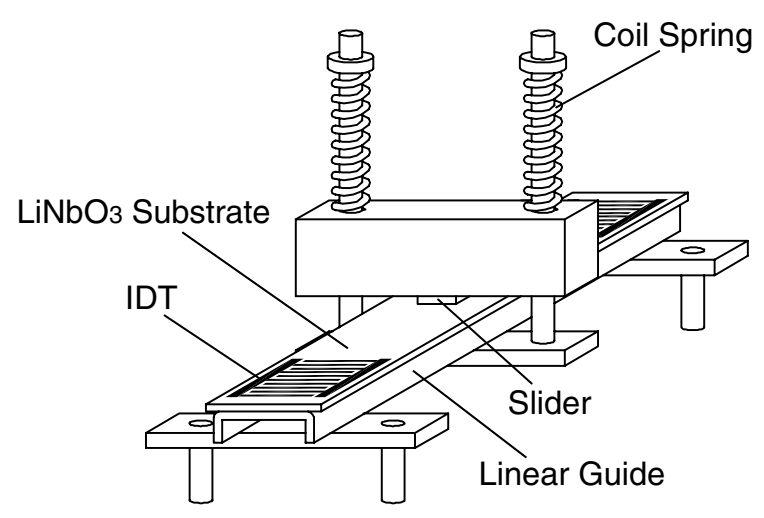

Fig. 1. Schema of a surface acoustic linear motor.

load and a friction coefficient, and the direction of the output force depends on the difference between slider velocity and vibration velocity of the stator substrate. Consequently, the calculated output force is increasing as the pre-load is increasing.

In this paper, the influence of the pre-load and vibration amplitude on maximum output force or no-load velocity is found out by using the fabricated SAW linear motor. Furthermore, a new simulation model which takes account of rigidity of tangential direction in a stator substrate surface is proposed, and this model is validated by comparing with experimental results.

\section{EXPERIMENTAL SET-UP}

In order to measure the driving performance variation with the pre-load, a SAW linear motor shown in Fig. 1 was fabricated. The fabricated SAW linear motor consists of a fixed part and a movable part. The fixed part comprises a linear guide rail and a stator substrate 


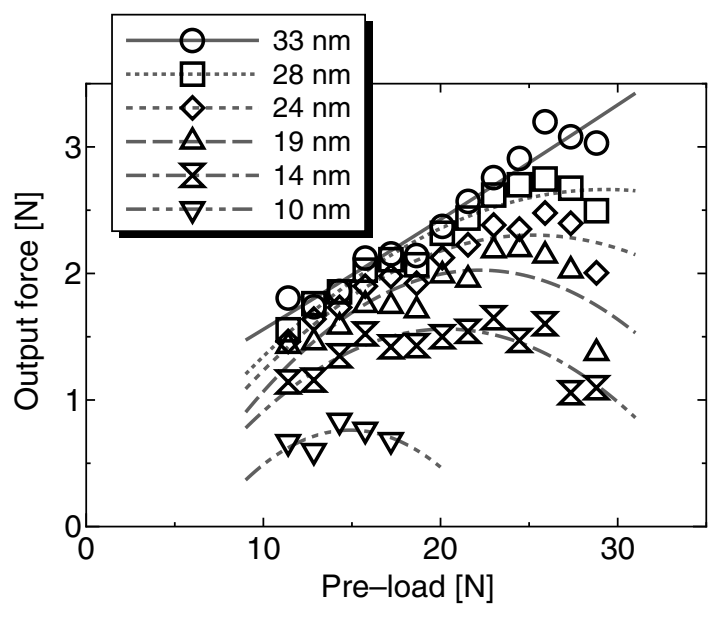

Fig. 2. Maximum output force against the pre-load about six kinds of vibration amplitude.

of 128 degrees rotated y-cut x-propagating $\mathrm{LiNbO}_{3}$. The movable part comprises a linear guide block, a silicon slider and two coil springs. The spring constant of the coil spring is $2.9 \mathrm{~N} / \mathrm{mm}$. The pre-load is adjusted up to $30 \mathrm{~N}$ by changing deflection of the coil springs. Mass of the movable part is $22.1 \mathrm{~g}$. The Rayleigh wave was excited by IDT with a high frequency electrical power source. The dimensions of the IDT were 400 $\mu \mathrm{m}$ in pitch, $100 \mu \mathrm{m}$ in electrode strip width and $9 \mathrm{~mm}$ in aperture size. The driving IDT is composed of 20 strip electrode pairs. The resonance frequency to propagate the Rayleigh wave is $9.61 \mathrm{MHz}$. Dimensions of the slider made of silicon are $4 \times 4 \times 0.3 \mathrm{~mm}^{3}$. There are many projections of pillar form on the contact surface of the silicon slider. The diameter of each projection is $20 \mu \mathrm{m}$. The projections are formed every $30 \mu \mathrm{m}$ directly by dry etching. The total number of the projections is 15,876 .

\section{EXPERIMENTAL RESULTS}

The driving performance was measured by using the fabricated SAW linear motor. The pre-load was changed from $11 \mathrm{~N}$ to $29 \mathrm{~N}$, and the normal vibration amplitude of the Rayleigh wave was changed from 10 $\mathrm{nm}$ to $33 \mathrm{~nm}$. The motion of movable part was observed by using a laser Doppler vibrometer. The maximum output force $F_{0}$ and no-load velocity $v_{0}$ were obtained from transient response.

Fig. 2 shows the maximum output force against the pre-load about six kinds of vibration amplitude. In this

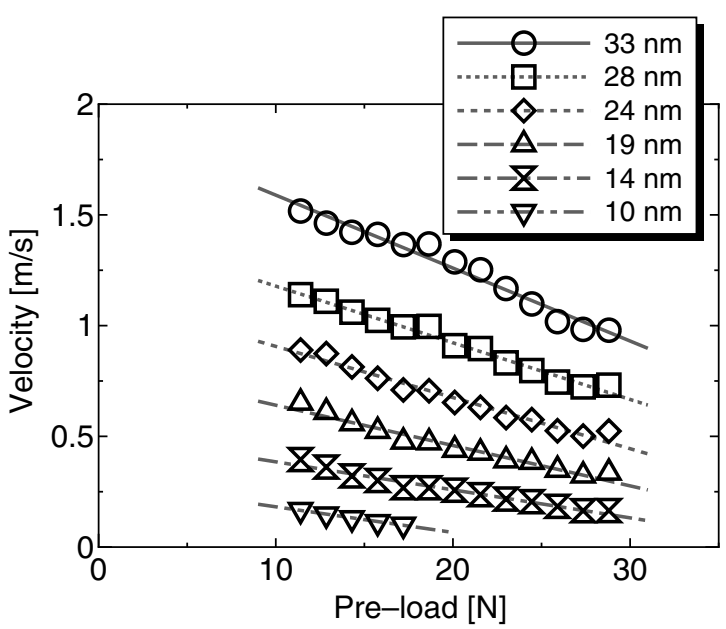

Fig. 3. No-load velocity against the pre-load about six kinds of vibration amplitude.

figure, similar to results obtained in the past researches, the optimum pre-load for the maximum output force existed. The optimum pre-load increased with increasing vibration amplitude. The maximum output force of $3.2 \mathrm{~N}$ was obtained at the pre-load of $26 \mathrm{~N}$ and the vibration amplitude of $33 \mathrm{~nm}$.

Fig. 3 shows the no-load velocity against the pre-load about six kinds of vibration amplitude. The no-load velocity decreased linearly with the pre-load. The noload velocity of $1.5 \mathrm{~m} / \mathrm{sec}$ was obtained at the pre-load of $11 \mathrm{~N}$ and the vibration amplitude of $33 \mathrm{~nm}$.

Fig. 4 shows the maximum output power against the pre-load about six kinds of vibration amplitude. The maximum output power $P_{\max }$ was calculated by the relation of $P_{\max }=v_{0} F_{0} / 4$ from the drooping forcevelocity characteristic. In this figure, the optimum preload for the maximum output power existed as well as the optimum pre-load for the maximum output force. The optimum pre-load for the maximum output power was less than the optimum pre-load for the maximum output force. This is because the no-load velocity increased with decreasing the pre-load. The maximum output power of $0.8 \mathrm{~W}$ was obtained at the vibration amplitude of $33 \mathrm{~nm}$.

The optimum pre-load against the vibration amplitude is shown in Fig. 5. The optimum pre-load adopted the value which makes the quadratic approximation function the maximum. In this figure, the optimum preloads for the maximum output force and for the maximum output power were both linear functions of the 


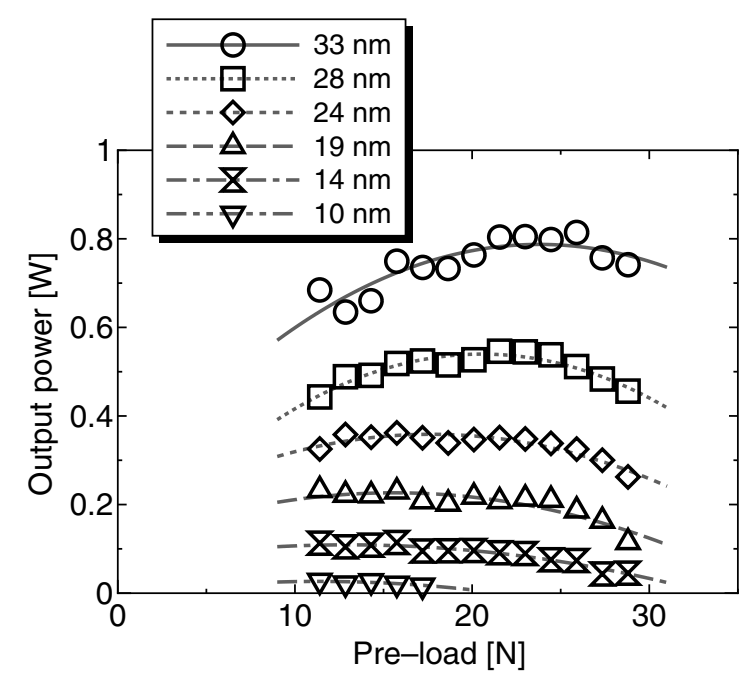

Fig. 4. Maximum output power against the pre-load about six kinds of vibration amplitude.

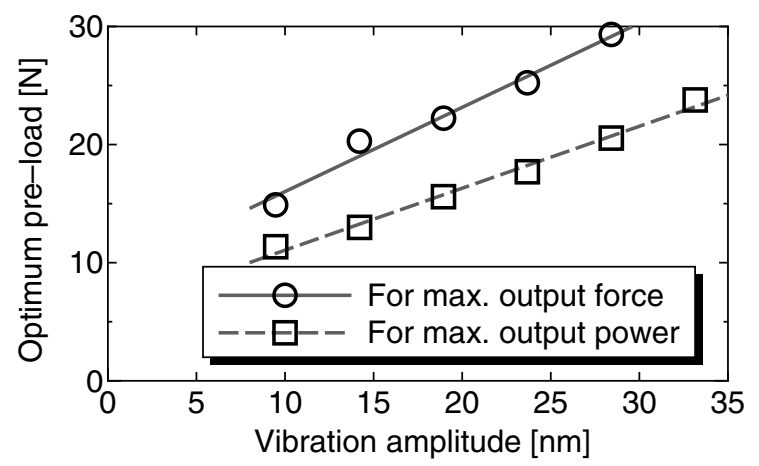

Fig. 5. Optimum pre-load against the vibration amplitude.

vibration amplitude. The ratio of the two optimum preloads was about 3:2.

\section{SimUlation MODEL}

In the new simulation model to propose, the rigidity of tangential direction in a stator substrate surface is taken into consideration. Therefore, the slipping state and the sticking state are distinguished. In the simulation model, since phase velocity of the Rayleigh wave is much faster than the slider velocity, the contact time of a projection and the stator substrate is assumed to be constant. Furthermore, since the stator deformation of tangential direction by the contact of projections is so small on comparison with the wavelength of the Rayleigh wave, effect of the deformation of tangential direction on the pre-load and the vibration displace- ment of normal direction can be assumed negligible. The output force of slider is the sum of the output force of each projection. There were assumptions as shown below for calculation.

- The diameter of projections is very small in comparison with the wavelength of the Rayleigh wave.

- Deformation of the stator substrate by one projection does not affect that by other projections.

- The projections are uniformly distributed to the phase of the Rayleigh wave.

Under such assumptions, the output force of slider is proportional to a time average of the output force of one projection. Therefore, only one projection is considered. The friction coefficient is assumed to be constant here.

During one cycle of the Rayleigh wave $(-\pi / 2 \leqq$ $\omega t \leqq 3 \pi / 2)$, normal vibration displacement is expressed as:

$$
y=a_{v} \sin \omega t
$$

where $a_{v}$ is the vibration amplitude of normal direction. At a critical point of $\omega t=\phi(-\pi / 2 \leqq \phi \leqq \pi / 2)$, the projection begins to contact the stator substrate, and stops contacting at $\omega t=\pi-\phi$. Therefore, the pre-load varies temporally as:

$$
f_{n}=k_{v} a_{v}(\sin \omega t-\sin \phi)
$$

where $k_{v}$ is the rigidity of normal direction in the stator substrate surface. On the other hand, tangential vibration displacement is expressed as:

$$
x=-a_{h} \cos \omega t
$$

where $a_{h}$ is the vibration amplitude of tangential direction. The temporal output force is proportional to the deformation of tangential direction at the contacting point of the projection. Therefore, the temporal output force $f$ is expressed as:

$$
\begin{aligned}
f & =k_{h}\left(x+a_{h} \cos \phi-\left(v t-a_{h} \phi \sin \psi\right)\right) \\
& =k_{h} a_{h}(\cos \phi-\cos \omega t-(\omega t-\phi) \sin \psi)
\end{aligned}
$$

where $k_{h}$ is the rigidity of tangential direction in the stator substrate surface and $\psi$ indicates the slider velocity $v: v=a_{h} \omega \sin \psi(-\pi / 2 \leqq \psi \leqq \pi / 2)$. If $|f| \leqq \mu f_{n}$, the projection sticks to the stator substrate surface. On the other hand, if $|f|>\mu f_{n}$, the projection slips and 


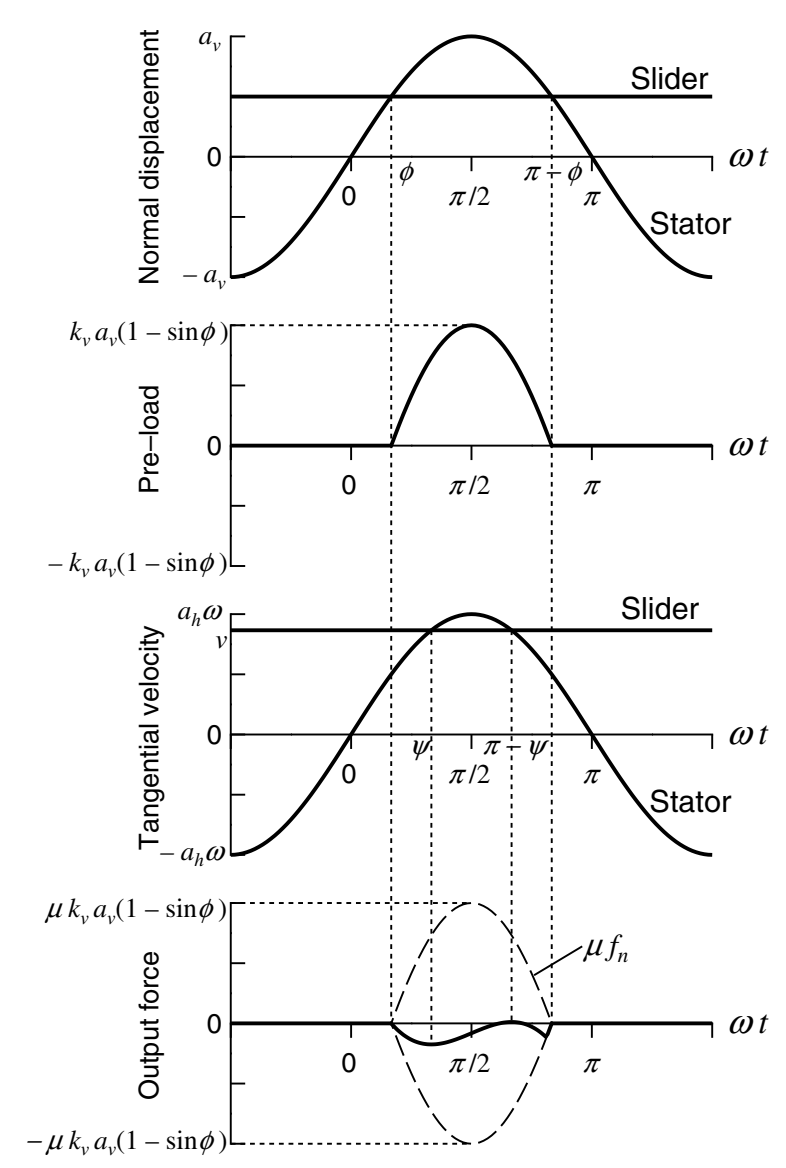

Fig. 6. Example of the time variation of normal displacement etc. that were calculated by the proposed simulation model.

the deformation of stator substrate decreases. In this case, the temporal output force $f_{\text {slip }}$ is expressed as:

$$
f_{\text {slip }}=\operatorname{sgn}(f) \cdot \mu f_{n} .
$$

As shown in equations (2) and (4), the stick/slip phenomena calculated by using this simulation model depend on three parameters, $\mu k_{v} a_{v} /\left(k_{h} a_{h}\right), \phi$, and $\psi$. The output force is calculated by judging stick/slip for every time step.

Fig. 6 shows an example of the time variation of normal displacement, the pre-load, velocity, and output force that were calculated by the proposed simulation model under $\mu k_{v} a_{v} /\left(k_{h} a_{h}\right)=1$. Fig. 7 shows the noload velocity and the maximum output force against the pre-load. In this figure, the no-load velocity $v_{0}$, the maximum output force $F_{0}$ and the pre-load $N$ are normalized by $v_{a}: v_{a}=a_{h} \omega, F_{a}: F_{a}=\mu k_{v} a_{v} /(2 \pi)$ and $N_{a}: N_{a}=k_{v} a_{v}$ respectively, and $\alpha=\mu k_{v} a_{v} /\left(k_{h} a_{h}\right)$.

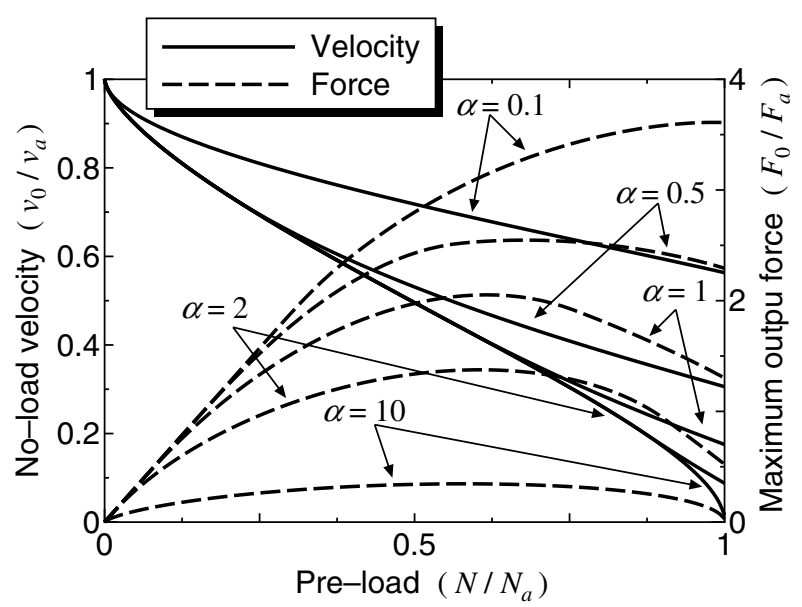

Fig. 7. No-load velocity and maximum output force against the pre-load.

The tendency of calculation results shown in Fig. 7 agrees with that of the experimental results. The optimum pre-load for the output force is well explained by using the simulation model.

\section{ESTIMATION}

The calculation results were compared with the experimental results in order to validate the simulation model. In the calculation, the friction coefficient $\mu$ between the silicon and the 128 degrees rotated y-cut $\mathrm{LiNbO}_{3}$ is 0.18 measured by a friction tester, and $k_{v}$ is $3.7 \times 10^{6} \mathrm{~N} / \mathrm{m}$ calculated from the formula of contact stress by a rigid punch, and $k_{v} / k_{h}$ is 1.36 calculated by using a finite element method, and $a_{v} / a_{h}$ is 1.12 from the result of numerical analysis. Therefore, $\alpha$ is 0.27 . The calculation results compared with the experimental results are shown in Fig. 8 and Fig. 9. Since each projection of the silicon slider is not held elastically, the contact state of the projections was not uniform. Therefore, the apparent friction coefficient, the maximum output force divided by the pre-load, was estimated instead of the maximum output force. The no-load velocity and the pre-load were normalized by the stator vibration velocity of tangential direction and the optimum pre-load for the maximum output force, respectively. In these figures, $F_{\mathrm{R}}$ denotes the resistance against driving the slider and $N_{\text {opt }}$ denotes the optimum pre-load for the maximum output force. The calculations were performed under $F_{\mathrm{R}}=0,0.02 N_{\mathrm{opt}}$, and $0.04 N_{\text {opt }}$. There is a good agreement between the ex- 


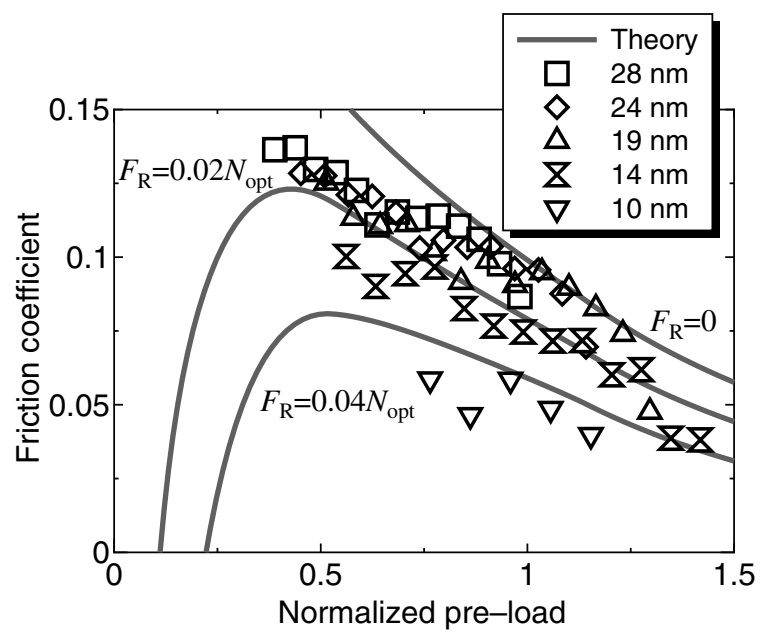

Fig. 8. Friction coefficient against the normalized preload

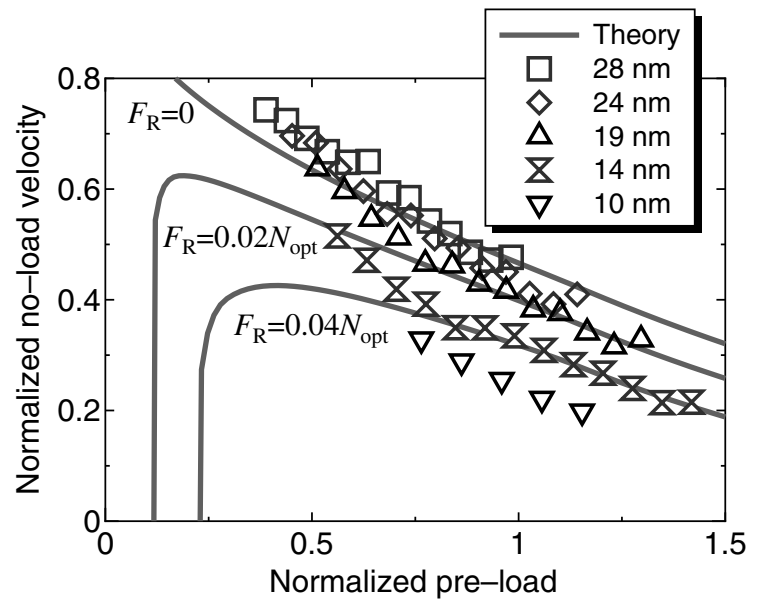

Fig. 9. Normalized no-load velocity against the normalized pre-load

perimental and simulation results, and the simulation model was validated.

In this figure, effect of the resistance against driving the slider is increasing when the vibration amplitude is decreasing. Therefore, this resistance is not so much dependent on the vibration amplitude. As the resistance against driving the slider, resistance by the variation in the contact condition of projections, resistance of the linear guide, resistance by the roughness of contact surface, etc. can be expected.

The optimum pre-load for the maximum output force at the vibration amplitude of $28 \mathrm{~nm}$ is $29 \mathrm{~N}$. This value is 314 times as large as the calculated optimum preload per one projection. The number of 314 is only $2 \%$ of the total number of projections. Furthermore, the maximum output force per unit contact area of 50-130 $\mathrm{N} / \mathrm{mm}^{2}$ was obtained by using the multi-contact-point slider of which the projections were held elastically [1]. On the other hand, the value of only $0.64 \mathrm{~N} / \mathrm{mm}^{2}$ was obtained by using the silicon slider from the present experimental result. Therefore, by improving the contact condition, the maximum output force per unit contact area is expected to be improved to $32 \mathrm{~N} / \mathrm{mm}^{2}$ which is 50 times higher than the present value.

\section{CONCLUSION}

The influence of the pre-load and vibration amplitude on maximum output force or no-load velocity was found out by using the fabricated SAW linear motor. The optimum pre-loads for the maximum output force and for the maximum output power were both linear functions of the SAW linear motor's vibration amplitude. Furthermore, a new simulation model which took account of rigidity of tangential direction in a stator substrate surface was proposed. It was enabled to calculate the optimum pre-load by using the simulation model. This model was validated by comparing with experimental results. From the calculation result, the maximum output force per unit contact area is expected to be improved to 50 times higher than the present value by improving the contact condition.

\section{ACKNOWLEDGMENTS}

The authors thank Dr. N. Shibaike of Matsushita Electric Industrial Co., Ltd. for valuable discussions.

\section{REFERENCES}

[1] M. K. Kurosawa, M. Chiba, and T. Higuchi, "Evaluation of a surface acoustic wave motor with a multi-contact-point slider," Smart Materials and Structures, vol.7, pp.305-311, 1998.

[2] N. Osakabe, M. Kurosawa, T. Higuchi, and O. Shinoura, "Surface acoustic wave linear motor using silicon slider," Proc. of IEEE Workshop on Micro Electro Mechanical Systems, pp. 390-395, 1998.

[3] M. Kurosawa, M. Takahashi, and T. Higuchi, " Contact and driving condition of a surface acoustic wave motor," Proc. of IFAC-IFIP-IMACS Conference on Control of Industrial Systems, pp. 396401, 1997. 